\title{
Early Correction of Rotated I ncisor Using 2x4 Appliance With Laser Aided Circumferential Supracrestal Fiberotomy
}

Radhika E, ${ }^{1}$ Rajendra Reddy E, ${ }^{2}$ Vinay kumar $L,{ }^{3}$ Thabitha Rani $S^{4}$

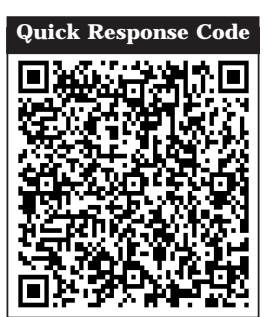

doi: $10.5866 / 2015.7 .10298$

${ }^{1}$ Post Graduate Student

2Professor \& HOD

${ }^{3}$ Senior Lecturer

${ }^{4}$ Professor

Department of Pedodontics \& Preventive Dentistry,

Kamineni Institute of Dental Sciences

Narketpally.

\section{Article Info:}

Received: October 10, 2015

Review Completed: November 11, 2015

Accepted: December 12, 2015

Available Online: March, 2016 (www.nacd.in)

C NAD, 2015 - All rights reserved

\section{Email for correspondence:}

drradhikabhm@gmail.com

\begin{abstract}
:
The tooth rotation is a dental anomaly of position, in which there is a displacement of the tooth within the alveolar bone around its longitudinal axis. The case presented in this article depicts the management of a rotated 22 by orthodontic derotation using $2 \times 4$ appliance and Circumferential supracrestal fiberotomy (CSF) with laser, followed by splinting with a bonded lingual retainer. This appliance comprised of bonds on the maxillary incisors, bands on the first permanent maxillary molars and a continuous arch wire. This appliance offers many advantages like complete control of anterior tooth position, is extremely well tolerated, requires no adjustment by the patient and allows accurate and rapid positioning of the teeth. After 4 months, the upper left lateral incisor was orthodontically brought into proper alignment. Circumferential supracrestal fiberotomy with laser was done next to the correction of the tooth, followed by bonded lingual retainer. Early treatment in these cases will not only quickly restore anterior aesthetics but may also reduce the future cumbersome orthodontic treatment.
\end{abstract}

Key words: Tooth rotation, 2x4 appliance, diode laser, bonded lingual retainer.

\section{INTRODUCTION:}

During the devel opment of the complex maxillomandibular structures, dental elements are likely to suffer from disorders caused by idiopathic, hereditary or environmental factors resulting in developmental abnormalities. ${ }^{1}$ The anomalies of tooth position among these disorders are: rotation of teeth, migration and transposition. ${ }^{2} \mathrm{~A}$ rotated incisor creates a considerable aesthetic and psychological problem for a patient. The rotation of permanent teeth can be due to pre, post-eruptive disturbances and because of retained deciduous teeth beyond normal exfoliation time. ${ }^{3,4}$

Complications involving the late treatment of a rotated permanent incisors includes dilacerations of the devel oping roots, root resorption, loss of tooth vitality, compromised oral hygiene. ${ }^{5,6} \mathrm{~A}$ significant delay in treatment can create the necessity for more complicated surgical and orthodontic management. The routine treatment of rotated teeth is use of fixed 
orthodontic appliance. The 2x4 fixed appliance comprises bands on the first permanent molars, bonds on the erupted maxillary permanent incisors and a continuous arch wire to provide complete control of the anterior dentition as well as a good archform. $^{7}$

Stability of rotated teeth is a concern in orthodontic treatment. A significant cause of relapse is thought to be the gingival and transseptal fibers of the periodontium, which are stretched and twisted as the tooth is rotated. ${ }^{8}$ To relieve the rotated tooth from forces exerted by the stretched fibers, CSF was introduced which appears to hel p thetissue remodel and decrease relapse of orthodontically rotated teeth. ${ }^{9}$

Retention is usually necessary following orthodontic treatment to overcome the elastic recoil of the periodontal supporting fibers and to allow remodeling of the alveolar bone. Bonded lingual retainers have been shown to be an effective means of retaining aligned anterior teeth in the posttreatment position in the long term. ${ }^{10}$

The case presented in this article delineates the management of a rotated upper left lateral incisor with orthodontic derotation by using $2 \times 4$ appliance, followed by laser assisted circumferential supracrestal fiberotomy and splinting with bonded maxillary custom lingual retainer.

\section{CASE REPORT:}

A 12 year old girl had reported to the OPD of the Department of Pedodontics and Preventive Dentistry of Kamineni Institute of Dental Sciences with the chief complaint of irregularly positioned upper front teeth. No significant history of trauma and medical history were found. Her facial profile was mild convex. The clinical examination showed good facial symmetry and competent lips at rest. Intra oral examination revealed Class I malocd usion and distopalatally rotated maxillary left lateral incisor with around 60^ rotation (Figure 1). Oral hygiene was poor and associated with mild gingivitis. After looking out for any other abnormality which was not found, choice of $2 \times 4$ appliance was made to correct the minor malocclusion. It engages both the maxillary first permanent molars, central incisors and lateral incisors in its set up.

The $2 \times 4$ appliance design: The basic $2 \times 4$ appliance design is as follows:
- Bands cemented on both maxillary permanent first molars

- Brackets bonded onto the erupted maxillary central and lateral incisors

- Continuous archwire of 0.016 inch nickel titanium to maintain good arch form, as well as control of anterior teeth.

Bracket was also bonded on lingual side of 22 to engage the $E$-chain, which was engaged from the lingual bracket of 22 to the labial brackets of 21,11 and 12 for the derotation of left lateral incisor and to close the spacing between right lateral and central incisors (Figure 2). The girl was seen for routine orthodontic adjustment visits every two weeks. After 4 months of these mechanics, the upper left lateral incisor was repositioned to its normal position (Figure 4). After correction of rotation, circumferential suprecrestal fiberotomy was performed using a diode laser (Figure 3), followed by placement of bonded retainers on the palatal surfaces of the maxillary incisors and canines. At 1 year follow-up, there were no pathologic findings in the radiographic view like root resorption, loss of lamina dura, widening of PDL and periapical radiolucency.

\section{DISCUSSION:}

The rotation, classified as one of the changes of position which was subjectively defined as an intraalveolar rotation mesiolingual or distolingual of the dental element around its long axis. Gupta et al. classified the rotation into three groups: $<45^{\circ}, 45-$ $90^{\circ}$ and $>90^{\circ}$. In his study, the majority of tooth rotations were between $45^{\circ}$ and $90^{\circ}$, foll owed by $<45^{\circ}$ rotations. ${ }^{11}$

Rotated teeth can be corrected by removable, semi-fixed or fixed appliance depending upon the severity of rotation. Disadvantages of removable appliances includes damage/loss of appliances, difficulty in speech/eating, gagging, decalcification/ caries, gingivitis/palatal hyperplasia/fungal infections, incorrect activation produces unhelpful changes, allow only tipping of teeth and it depends on patient compliance. Advantages of fixed appliances includes minimal discomfort, reduces need for patient co-operation, increase control of tooth movements and movement possiblein all three planes of space. ${ }^{12}$ The $2 \times 4$ partial fixed appliance offers more effective and efficient tooth positioning 


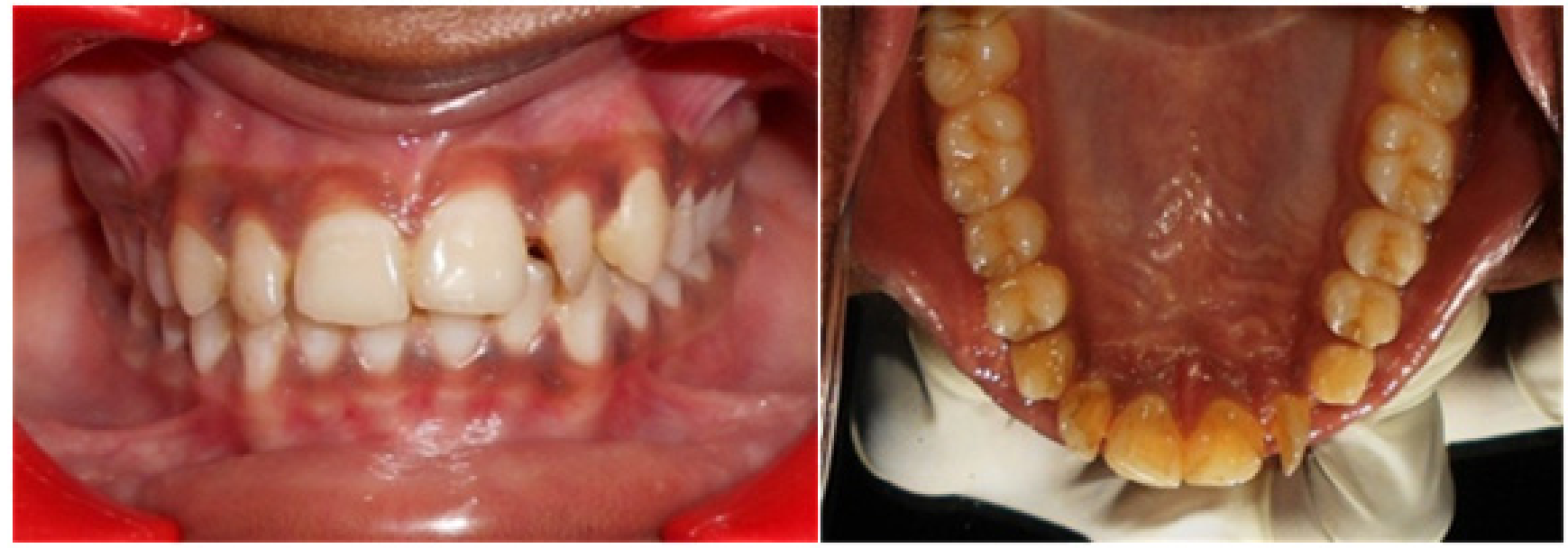

Figure 1: Pre-operative picture showing distopalatally rotated maxillary left lateral incisor with around 60? rotation.

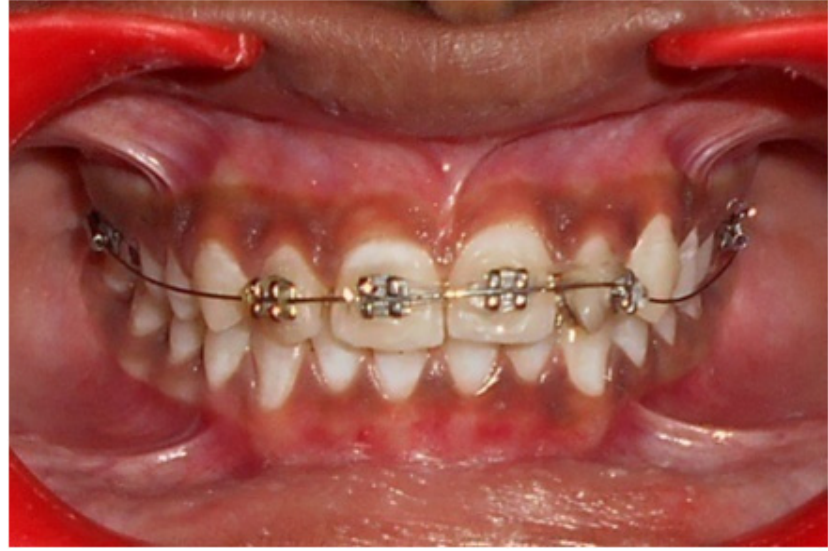

Figure 2: E-chain engaged to 12, 11, 21 and 22

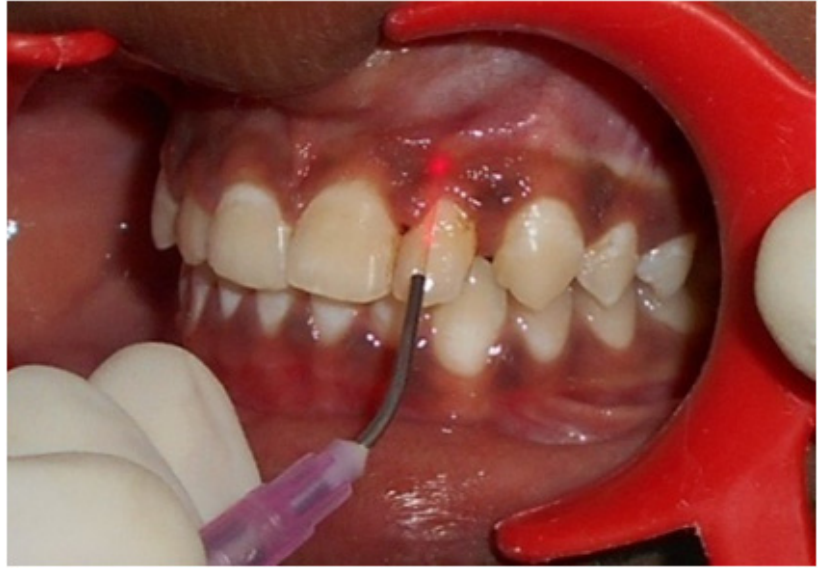

Figure 3: Circumferential supracrestal fiberotomy with Laser

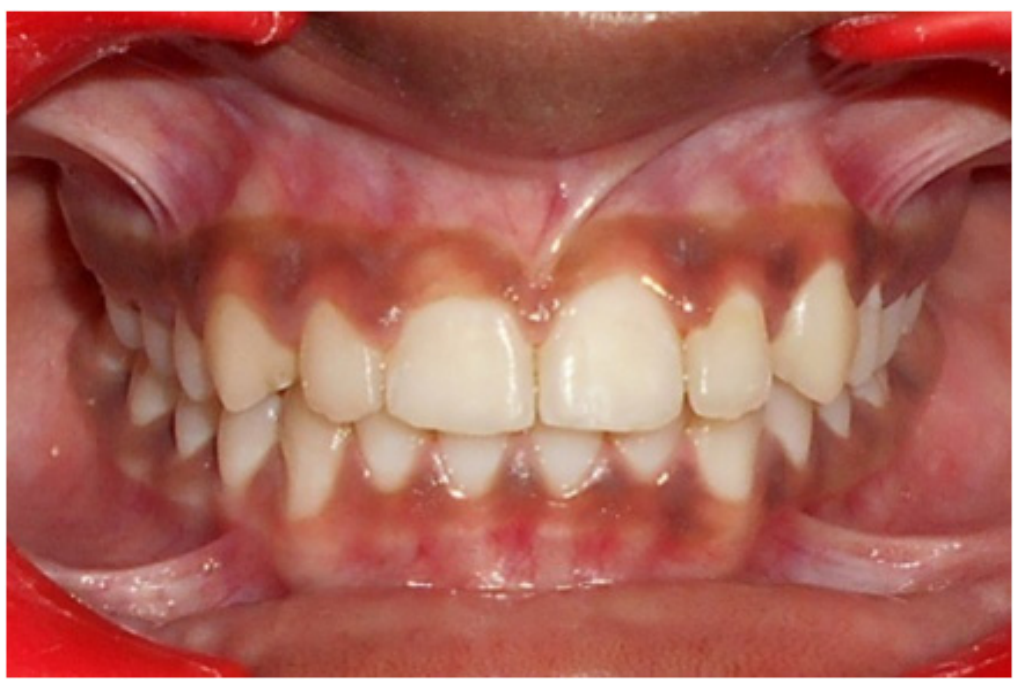

Figure 4: The upper left lateral incisor was repositioned to its normal position 
as it allows three dimensional control of the involved teeth. Rotations, diastemas, incorrect tooth inclinations and angulations may therefore be treated very quickly using this versatile technique. ${ }^{7}$

Relapse of tooth position after orthodontic correction has always been a great challenge. It has been proposed that orthodontic movement to correct rotated teeth results in stretching of the collagen fibers and might be implicated in rotational relapse by pulling the teeth back toward their pretreatment position. This is due to considerable residual forces which remain in the tissues of the periodontium after tooth movement. ${ }^{13}$ Circumferential supracrestal fiberotomy was advocated for the release of soft tissue tension and reattachment of the fibers after orthodontic correction of tooth rotation. Conventional CSF with scalpel blade has some clinical drawbacks: poor patient acceptability as an invasive procedure, and it is feasible only on teeth with a healthy periodontium. ${ }^{14}$ Laser offers numerous advantages compared with surgery, it stimulates wound healing, collagen synthesis, bone remodeling, coagulates blood vessels, seals lymphatics and sterilizes the wound during ablation while maintaining a clear, clean, surgical field with markedly less bleeding, minimal swelling, and less postoperative infection. Laser CSF is expected to prevent relapse of orthodontically rotated teeth in addition to the advantages offered by the properties of laser. 15

It has been advocated that the retention of tooth movement is mandatory at the end of an active orthodontic treatment, due to potential unstable tooth position. A multi-stranded wire individually adjusted and bonded to each tooth in the desired arch segment was used for long-term retention. 2x4 appliance was considered to be reliable, independent of patient compliance, highly effective, relatively easy to apply, almost invisible and well accepted by patient.

\section{CONCLUSION:}

The present case demonstrated the versatility of the $2 \times 4$ appliance in the correction of rotation and alignment of the incisors. The derotation of 22 was achieved within a short duration of time and alignment was maintained by performing laser assisted CSF, followed by bonded retainer. Early treatment in these cases will not only quickly restore anterior aesthetics but may also reduce the extensive orthodontic treatment at a later stage. The functional improvement coupled with the psychological benefit gives this simple and easily placed appliance a significant advantage over the traditional methods.

\section{REFERENCES:}

1. Teixeira VP, Martins MAT, Lascala CA, Marques MM, Rossi, J M, Missawa GTM, Martins MD. Study dental abnormalities development in patients undergoing orthodontic treatment. J Inst Health Sci 2008; 26(4):454457.

2. Pedreira EN, Magalhaes MC, Cardoso CL, Taveira LA, de Freitas CF. Radiographic study of dental anomalies in Brazilian patients with neuropsychomotor disorders. J Appl Oral Scl 2007; 15(6):524-528.

3. Kim YH, ShiereFR, Fogels HR. Pre-eruptive factors of tooth rotation and axial inclination. J Dent Res 1961; 40:548-557.

4. Morgan G. E. Prolonged Retention: When Should Healthy Deciduous Teeth Be Extracted? J Am Dent Assoc 1938; 25:358.

5. Giancotti A, Grazzini F, DeDominicis F, Romanini G, Arcuri C. Multidisciplinary evaluation and clinical management of mesiodens. J Clin Pediatr Dent 2002; 26(3):233-237.

6. Gunduz K, Muglali M. Non-syndrome multiple supernumerary teeth: A case report. J Contemp Dent Pract 2007; 8(4):81-87.

7. McKeown HF, Sandler J. The two by four appliance: a versatile appliance. Dent U pdate 2001; 28(10):496-500.

8. Thompson HE. Orthodontic relapses analyzed in a study of connective tissue fibers. Am J Orthod. 1959; 45:93-109.

9. Edwards J G. A surgical procedure to eliminate rotational relapse. Am J Orthod 1970; 57(1):35-46.

10. Butler J, Dowling P. Orthodontic bonded retainers. J Ir Dent Assoc 2005; 51(1):29-32.

11. Gupta SK, Saxena P, J ain S, J ain D. Prevalence and distribution of selected developmental dental anomalies in an Indian population. J Oral Sci 2011; 53(2):231-238.

12. Dowsing $P$, Sandler PJ. How to effectively use a $2 \times 4$ appliance. J Orthod 2004; 31(3):248-258.

13. Reitan $K$. Tissue rearrangement during retention of orthodontically rotated teeth. Angle Orthod 1959; 29:105113.

14. Kim SJ, Paek J H, Park KH, Kang SG, Park YG. Laser-aided circumferential supracrestal fiberotomy and low level laser therapy effects on relapse of rotatedteeth in beagles. Angle Orthod 2010; 80(2):385-390.

15. Hall RR. The healing of tissues incised by a carbon-dioxide laser. Br J Surg 1971; 58(3):222-225. 